\title{
Dipole-exchange spin waves in a periodically layered ferromagnetic nanotube
}

\author{
Y.I.Gorobets, V.V.Kulish* \\ Institute of Magnetism, National Academy of Sciences of Ukraine, \\ 36-b Vernadskogo Str., 03142 Kyiv, Ukraine \\ "Department of General and Experimental Physics, National Technical \\ University of Ukraine "Kyiv Polytechnic Institute", \\ 37 Peremogy Ave., 03056 Kyiv, Ukraine
}

\section{Received August 11, 2013}

\begin{abstract}
Spin waves in a periodically layered ferromagnetic nanotube (nanotube magnetophotonic crystal) are investigated. External magnetic field is considered to be applied parallel to the nanotube symmetry axis. The linearized Landau-Lifshitz equation in magnetostatic approximation is used, taking into account the magnetic dipole-dipole interaction, theexchange interaction and the anisotropy effects. As a result, the local dispersion relation (for uniform nanotube sections), the radial wave number spectrum and the longitudinal quasiwave number spectrum (for the entire nanotube) for spin waves in the above-described nanotube are found. From the radial wave number spectrum, limitations on the transverseangular modes are defined. The longitudinal quasi-wave number spectrum in the "effective medium" limit is shown to have the same form as for a uniform nanotube (with averaged parameters).
\end{abstract}

Исследованы спиновые волны в периодически-слоистых ферромагнитных нанотрубках (магнитофотонный кристалл-нанотрубка). Внешнее магнитное поле считается параллельным оси симметрии нанотрубки. Использовано линеаризованное уравнение Ландау-Лифшица в магнитостатическом приближении с учетом магнитного диполь-дипольного взаимодействия, обменного взаимодействия и әффектов анизотропии. В результате получено локальное дисперсионное отношение (для однородных секций нанотрубки), спектр радиальных волновых чисел и продольных квази-волновых чисел (для всей нанотрубки) для спиновых волн в вышеописанной нанотрубке. Из спектра радиальных волновых чисел определены ограничения на поперечно-угловые моды. Показано, что квази-волновое число в приближении "эффективной среды" имеет такой же вид, как и для однородной нанотрубки (с усредненными параметрами).

Дипольно-обмінні спінові хвилі у періодично-шаруватій феромагнітній нанотрубиі. Ю.І.Горобець, В.В.Куліш.

Досліджено спінові хвилі у періодично-шаруватих феромагнітних нанотрубках (магнітофотонний кристал-нанотрубка). Зовнішнє магнітне поле вважається паралельним до осі симетрії нанотрубки. Використовується лінеаризоване рівняння ЛандауЛіфшиця у магнітостатичному наближенні з урахуванням магнітної диполь-дипольної взаємодії, обмінної взаємодії та ефектів анізотропії. В результаті отримано локальне дисперсійне відношення (для однорідних секцій нанотрубки), спектр радіальних хвильових чисел та поздовжніх квазі-хвильових чисел (для всієї нанотрубки) для спінових хвиль у вищеописаній нанотрубці. Зі спектра радіальних хвильових чисел визначено обмеження на поперечно-кутові моди. Показано, що квазі-хвильове число у наближенні "ефективного середовища" має такий же вигляд, як і для однорідної нанотрубки (з усередненими параметрами). 


\section{Introduction}

In recent years, researchers of spin waves (magnetization excitations in magnetically ordered materials [1]), pay special attention to spin waves in nanoscale systems. Spin waves in thin ferromagnetic films [2-4], micron-sized magnetic quantum dots [5-7], nanowires [8-11] and other nanosystems are of special interest for practical applications (for instance, in new magnetic storage devices [12-14]).

Spin waves characteristics are known to depend strongly on the system shape and size. Therefore, it is necessary to investigate spin waves in different kinds of systems individually. In particular, synthesized recently metal nanotubes (including carbonic nanotubes or dielectric nanowires with metal shells $[15,16]$, nanotubes embedded in porous template [17] and so on) comprised of magnetic material [17-22] are of special interest to study. They have found a wide range of applications, particularly in magnetobiology [23, 24]. Still, little attention has been devoted to the spin waves in magnetic nanotubes, and known papers on this subject investigate mostly spin solitons [25] and waves on magnetic domains interfaces [26].

It is known that periodic nanoscale magnetic composites - magnetophotonic crystals [27] - exhibit anomalous optical and magneto-optical properties [27-29]. They are of particular interest for practical applications, mostly in magneto-optical devices (optical isolation devices [30], magnetooptic spatial light modulators [27] and so on). In particular, spin waves in such systems are studied intensively [31-35]. Therefore, study of spin waves in nanotube magnetophotonic crystals (periodically modulated magnetic nanotubes) is an actual and promising area of the physics of nanostructures.

In this article, we studied spin waves in a periodically layered (in the axial direction) ferromagnetic nanotube. For spin waves in such structure we have found a dispersion relation considering the magnetic dipole-dipole interaction, the exchange interaction and the anisotropy effects. We have also found the radial wave number and the longitudinal quasi-wave number spectrum for such waves.

\section{Setting of the problem}

Let us consider a periodically-structured cylindrical nanotube composed of alternat-

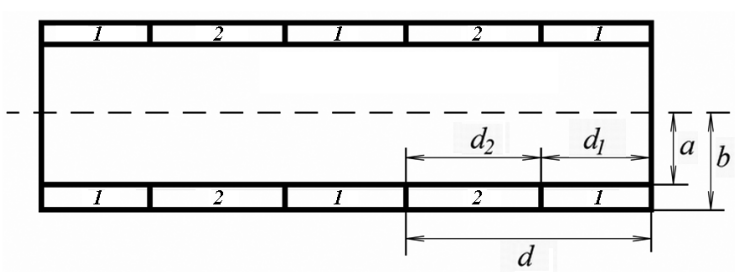

Fig. 1. Nanotube modeled in the article.

ing homogeneous sections consisting of two kinds of ferromagnetic, denoted 1 and 2 . Each section of one kind of ferromagnetic has the same length ( $d_{1}$ for the ferromagnetic $1, d_{2}$ for the ferromagnetic 2) and alternate, forming periodic structure with the period $d$. The medium inside and outside the nanotube is considered as non-magnetic. Inner radius of the nanotube is denoted $a$, external $-b$ (Fig. 1 ).

We consider the nanotube ferromagnetics that both have uniaxial magnetic anisotropy, with the magnetic anisotropy axis directed along the nanotube axis. Both ferromagnetics are considered to have "easy axis" type, so that the saturation magnetization is also directed along the symmetry axis of the nanotube. We assume that the ferromagnetics are characterized by the following parameters: the uniaxial anisotropy parameters $\beta_{1}$ and $\beta_{2}$ (are considered constant for each section), the exchange interaction parameters $\alpha_{1}$ and $\alpha_{2}$ (an exchange energy tensor in the general case of uniaxial crystal is diagonal and has two independent components; we consider the case when these components are equal - that is true for a cubic crystal, for a polycrystalline ferromagnetic with small crystals, etc.). We assume that saturation magnetization $\mathbf{M}_{0}$ is constant throughout the nanotube volume and is directed along the nanotube axis. We neglect the dissipation and, therefore, spin waves damping in the nanotube, discarding the relaxation terms in the Landau-Lifshitz equation. The gyromagnetic ratio $\left(\gamma_{1}\right.$ for the ferromagnetic $1, \gamma_{2}$ for the ferromagnetic 2 ) is considered constant.

Let us consider a spin wave propagating in the nanotube described above parallel to its axis. The spin wave is considered to be small perturbation of magnetization density and, correspondingly, of the magnetic field. Thus, perturbation $\mathbf{m}$ of the magnetization density $\left(\mathbf{M}=\mathbf{M}_{0}+\mathbf{m}\right.$, where $\mathbf{M}$ is the overall magnetization) must satisfy the condition $|\mathbf{m}|<<\| \mathbf{M}_{0} \mid$.

In this work, dispersion relation, radial wave number spectrum and longitudinal 
quasi-wave number spectrum of the abovedescribed spin waves is derived.

\section{Theoretical background}

Let us write down the Landau-Lifshitz equation for the nanotube described in the previous section.

We assume that deviations of the magnetization $\mathbf{m}$ and of the magnetic field $\mathbf{h}$ inside the ferromagnetic from their values in the ground state $-\mathbf{M}_{0}$ and $\boldsymbol{H}_{\mathbf{0}}^{(i)}$, respectively - are small, therefore we can use linearized form of the Landau-Lifshitz equation. Let us direct an axis $\mathrm{Oz}$ along the symmetry axis of the system. Then, the saturation magnetization and (because of our system symmetry) the ground state magnetic field inside each of the ferromagnetic sections are directed along the anisotropy axis of the system and, consequently, along the axis $\mathrm{Oz}$. So, for $\mathbf{m}$ and $\mathbf{h}$ written in the periodic by time form

$$
\begin{gathered}
\boldsymbol{m}(\boldsymbol{r}, t)=\boldsymbol{m}_{\mathbf{0}}(\boldsymbol{r}) \exp (i \omega t), \\
\boldsymbol{h}(\boldsymbol{r}, t)=\boldsymbol{h}_{\mathbf{0}}(\boldsymbol{r}) \exp (i \omega t)
\end{gathered}
$$

we, after omitting the damping term, obtain the linearized Landau-Lifshitz equation (see, e.g., [36]) in the form

$$
\begin{gathered}
i \omega \boldsymbol{m}_{\mathbf{0}}= \\
=\gamma_{j}\left(M_{\mathbf{0}} \boldsymbol{e}_{\boldsymbol{z}} \times\left(\boldsymbol{h}_{\mathbf{0}}+\alpha_{j} \sum_{i} \frac{\partial^{2} \boldsymbol{m}_{\mathbf{0}}}{\partial x_{i}^{2}}-\left(\beta_{j}+\frac{\boldsymbol{H}_{\mathbf{0}}^{(\boldsymbol{e})}}{M_{\mathbf{0}}}\right) \boldsymbol{m}_{\mathbf{0}}\right)\right),
\end{gathered}
$$

here $\boldsymbol{H}_{\boldsymbol{O}}^{(e)}$ is an external field (outside the nanotube), $j=1,2$ denotes sections of the ferromagnetic 1 and 2 , respectively, $\mathbf{e}_{z}$ is a unit vector of the axis $\mathrm{Oz}$.

In order to solve the Landau-Lifshitz equation we need one more relation between magnetization and magnetic field. Let us use the magnetostatic approximation [36] for spin waves in the nanotube. In this approximation, field deviation $\mathbf{h}$ is a potential field so that $\boldsymbol{h}=-\nabla \Phi, \mathbf{h}_{0}=-\nabla \Phi_{0}$, where $\Phi$ is potential and $\Phi=\Phi_{0}(\mathbf{r}) \exp (i \omega t)$. Then, from the Maxwell equation $\operatorname{div} \boldsymbol{h}=-4 \pi \cdot \operatorname{div} \boldsymbol{m}$ we obtain the sought relation:

$$
\Delta \Phi-4 \pi \operatorname{div} \boldsymbol{m}=0 .
$$

Equations (2) and (3) give us necessary relationship between $\mathbf{m}$ and $\mathbf{h}$. Using this equations system, we can find the dispersion relation and the wave number spectrum inside each of the nanotube sections.

\subsection{Dispersion relation and radial wave number spectrum}

Let us find the dispersion relation for spin waves on the homogeneous sections of the nanotube, using equation system (2), (3).

The system of equations (2), (3) (after taking into account $m_{0 z}=0$ ) after elimination of magnetization perturbation $\mathbf{m}$ can be reduced to the following equation for magnetic potential:

$$
\begin{aligned}
& \left(\frac{\omega^{2}}{\gamma_{j}^{2} M_{0}^{2}}-\left(\frac{H_{0}^{(e)}}{M_{0}}+\beta_{j}-\alpha_{j} \Delta\right) \times\right. \\
\times & \left.\left(\left(\frac{H_{0}^{(e)}}{M_{0}}+\beta_{j}\right)+4 \pi-\alpha_{j} \Delta\right)\right) \Delta \Phi_{0}+ \\
& +4 \pi\left(\frac{H_{0}^{(e)}}{M_{0}}+\beta_{j}-\alpha_{j} \Delta\right) \frac{\partial^{2} \Phi_{0}}{\partial z^{2}}=0 .
\end{aligned}
$$

Let us use the cylindrical coordinate system $(\rho, \theta, z)$. Then, equation (4) has solutions in the form

$\Phi=$

$=\left(A_{J} J_{n}\left(k_{\perp} \rho\right)+A_{N} N_{n}\left(k_{\perp} \rho\right) \exp \left(i\left(n \theta+k_{j \|} z-\omega t\right)\right)\right.$,

here $A_{J}$ and $A_{N}$ are constants, $J_{n}\left(k_{\perp} \rho\right)$ is the Bessel function, $N_{n}\left(k_{\perp} \rho\right)$ is the Neumann function, $k_{\perp}$ is a transverse wave number, $n$ is a transverse-angular mode number; $k_{\perp}$ and $n$ are equal along all the nanotube sections. (This form of the solution becomes evident if we write down the relation $\Delta \Phi=-\left(k_{\perp}^{2}+k_{j}^{2}\right) \Phi$ for the potential given by

(5)). After substituting the solution (5) into equation (4) we obtain dispersion equation in the form

$$
\begin{gathered}
\left.\alpha_{j}^{2}\left(k_{j \mid}^{2}+k_{\perp}^{2}\right)^{3}+2 \alpha_{j}\left(\tilde{\beta}_{j}+2 \pi\right)\left(k_{j \mid}^{2}+k_{\perp}^{2}\right)^{2}\right)+ \\
\left(\widetilde{\beta}_{j}\left(\widetilde{\beta}_{j}+4 \pi\right)-\frac{\omega^{2}}{\gamma_{j}^{2} M_{0}^{2}}-4 \pi \alpha_{j} k_{j \|}^{2}\right)\left(k_{j||}^{2}+k_{\perp}^{2}\right)- \\
-4 \pi \widetilde{\beta}_{j} k_{j \mid}^{2}=0,
\end{gathered}
$$

where $\tilde{\beta}_{j}=\frac{H_{0}^{(e)}}{M_{0}}+\beta_{j}$. This equation corresponds to the following a dispersion relation:

$=\gamma_{j} M_{0}{\sqrt{\alpha_{j}^{2} k_{j}^{4}+2 \alpha_{j}\left(2 \pi+\tilde{\beta}_{)} k_{j}^{2}+\tilde{\beta}_{j}\left(4 \pi+\widetilde{\beta}_{j}\right)-4 \pi k_{j(}^{2}\left(\alpha_{j}+\frac{\beta_{j}}{k_{j}^{2}}\right)\right.}}^{(8)}$ 
where the total wave number $k_{j}^{2}=k_{j}^{2}+k^{2}$. Note that the result obtained matches the dispersion relation obtained in [8, 31] for cylindrical nanowires. Thus, the transition from nanowires to nanotubes does not change the spin waves pattern in the system.

Let us find the transverse wave number spectrum. We can do this by imposing the boundary conditions for the magnetization perturbation on the outer border of the nanotube. In the absence of magnetic moment outside the nanotube we obtain $\left.\mathbf{m}\right|_{\rho=a, b}=0,\left.\quad \frac{\partial m}{\partial \rho}\right|_{\rho=a, b}=0$. Maxwell equation (4) allows us to rewrite these boundary conditions for the symmetry of our system as $\left.\Delta \Phi\right|_{\rho=a, b}=0$. From the other side, the condition $\left.\Delta \Phi\right|_{\rho=a, b}=0$ can be rewritten as $\left.k_{j}^{2} \Phi_{0}\right|_{a, b}=0$ after using the following property of the Bessel functions

$$
\frac{1 d}{\rho d \rho} \rho \frac{d}{d \rho} \Phi_{0}=\left(-k_{\perp}^{2}+\frac{n^{2}}{\rho^{2}}\right) \Phi_{0} .
$$

So, for $k_{j} \neq 0$ we can rewrite $\left.\Delta \Phi\right|_{\rho=a, b}=0$ as $\left.\Phi_{0}\right|_{a, b}$, or

$$
\begin{gathered}
A_{J} J_{n}\left(k_{\perp} a\right)+A_{N} N_{n}\left(k_{\perp} a\right)= \\
=A_{J} J_{n}\left(k_{\perp} b\right)+A_{N} N_{n}\left(k_{\perp} b\right)=0 .
\end{gathered}
$$

For a wide nanotube, so that $k_{\perp} a>1$, or for a nanotube that is thin compared to its width, so that $b-a / a<<1$, the expression for the transverse wave number spectrum (10) can be simplified significantly. (Since $k_{\perp} a$ is of the same order of magnitude or greater than $1 /(b-a)$, for thin nanotubes, the condition $k_{\perp} a>1$ is satisfied). Since for such nanotubes we can use the Bessel functions asymptotics and rewrite

$$
\Phi_{0}(\boldsymbol{r})=\frac{C}{\sqrt{r}} \sin (k r+\delta) \exp \left(i\left(n \theta+k_{j||} z\right)\right),
$$

where $C$ is a normalization constant and $\delta$ is an initial phase, from the boundary conditions (10) we can obtain $k_{\perp}$ in the form

$$
k_{\perp}=\frac{\pi n}{b-a} .
$$

The transverse wave number spectrum for thin nanotube (11) is analogous to the spectrum for a particle in one-dimensional potential well, so the problem becomes quasi-one-dimensional for the thin nano- tube. Note that the thin nanotube condition $b-a / a<<1$ that allows for the transverse wave number to be written in the simplified form (11) is satisfied for typical nanotubes.

\subsection{Wave number spectrum in the entire nanotube}

Let us find an effective longitudinal quasi-wave number spectrum in the entire nanotube. To do this, we - similarly to the method used in [31, 37] - apply the spatial periodicity condition (the Bloch theorem) to the spin waves in the nanotube. According to this theorem, the quasi-wave number $K$ must satisfy the relation

$$
\boldsymbol{m}(z)=\exp (i K d) \boldsymbol{m}(z+d) .
$$

After applying the Bloch theorem and the boundary conditions on the layers interfaces $z_{m}$

$$
\left.\boldsymbol{m}\right|_{z_{m}+0}=\left.\boldsymbol{m}\right|_{z_{m}-0},\left.\quad \alpha \frac{\partial \boldsymbol{m}}{\partial z}\right|_{z_{m}+0}=\left.\alpha \frac{\partial \boldsymbol{m}}{\partial z}\right|_{z_{m}-0}
$$

to a superposition of direct and reflected traveling spin waves

$$
\boldsymbol{m}_{\mathbf{0}}=\boldsymbol{C}_{\mathbf{1}}(\rho, \theta) \exp (i K z)+\boldsymbol{C}_{\mathbf{2}}(\rho, \theta) \exp (-i K z)
$$

we obtain an expression for the quasi-wave number of the spin wave in the nanotube that is analogous to the expression obtained in $[31,37]$ for a periodically-structured nanowire and a multilayer film:

$$
\begin{gathered}
\cos (K d)=\cos \left(k_{1 \|} d_{1}\right) \cos \left(k_{2} d_{2}\right)- \\
-\frac{1}{2}\left(\frac{\alpha_{2} k_{2} \|}{\alpha_{1} k_{1 \|}}+\frac{\alpha_{1} k_{1} \|}{\alpha_{2} k_{2 \|}}\right) \sin \left(k_{1 \|} d_{1}\right) \sin \left(k_{2 \|} d_{2}\right) .
\end{gathered}
$$
ber

Hence we obtain for the quasi-wave num-

$$
\begin{aligned}
& K=\arccos \left(\frac { 1 } { d } \left(\cos \left(k_{1 \|} d_{1}\right) \cos \left(k_{2} d_{2}\right)-\right.\right. \\
& \left.-\frac{1}{2}\left(\frac{\alpha_{2} k_{2 \|}}{\alpha_{1} k_{1} \|}+\frac{\alpha_{1} k_{1 \|}}{\alpha_{2} k_{2 \|}}\right) \sin \left(k_{1 \|} d_{1}\right) \sin \left(k_{2 \|} d_{2}\right)\right),
\end{aligned}
$$

where the local longitudinal wave numbers $k_{1 \|}, k_{2 \|}$ can be derived from the dispersion relation $(8)$.

More simple and physically clear expression for the effective wave number can be obtained from expression (16) in the "effective medium" limit, see [2, 37]. In this approximation, the spin wave wavelength is much greater than the characteristic size of the medium inhomogeneities, in our case - 
of the system spatial period: $k_{j \|} d<<1$, $K d<<1$. In this limit the medium can be considered as quasi-one-dimensional, and the effective - average - parameters are used for its description. Thus, using the conditions $k_{j \|} d<<1, K d<<1$, we can rewrite (16) in the form

$$
\begin{aligned}
& \frac{(K d)^{2}}{2}=\frac{\left(k_{1} \| d_{1}\right)^{2}}{2}+\frac{\left(k_{2} \| d_{2}\right)^{2}}{2}+ \\
& +\frac{1}{2}\left(\frac{\alpha_{2} k_{2 \|}}{\alpha_{1} k_{1 \|}}+\frac{\alpha_{1} k_{1 \|}}{\alpha_{2} k_{2 \|}}\right) k_{1 \|} d_{1} k_{2 \|} d_{2}
\end{aligned}
$$

or after obvious transformations

$$
K=\sqrt{\frac{1}{\bar{\alpha}} \frac{k_{1 \|}^{2} d_{1} \alpha_{1}+k_{2||}^{2} d_{2} \alpha_{2}}{d_{1}+d_{2}}}=\frac{\sqrt{k^{2} \alpha}}{\bar{\alpha}},
$$

here $\bar{\alpha}=\left(\frac{\alpha_{1}^{-1} d_{1}+\alpha_{2}^{-1} d_{2}}{d_{1}+d_{2}}\right)^{-1}$,

$\overline{k^{2} \alpha}=\frac{k_{1 \|}^{2} d_{1} \alpha_{1}+k_{2 \|}^{2} d_{2} \alpha_{2}}{d_{1}+d_{2}},-$ effective aver-

aged parameters of the ferromagnetic nanotube.

\section{Discussion}

So far, we obtained local dispersion relation (given by (8)) for spin wave on the homogeneous nanotube sections as well as the transverse wave number spectrum (given by (10), (11)) and longitudinal quasiwave number spectrum (given by (19)) for spin wave in the entire periodically layered nanotube. Note that if the nanotube is thin (thickness $b-a$ is small compared to the characteristic length of the exchange interaction: $\left.b-a<<\mid l_{e x}\right)$, so that we can put $k_{\perp}=0$

(the magnetization is considered uniform along the nanotube thickness), the local dispersion relation (8) transforms as follows:

$$
\begin{gathered}
\omega=\gamma_{j} M_{0}\left(\alpha_{j} k_{j \mid}^{2}+\frac{H_{0}^{(e)}}{M_{0}}+\beta_{j}\right) \leftrightarrow \\
\leftrightarrow k_{j \|}=\sqrt{\frac{1}{\alpha_{j}}\left(\frac{\omega}{\gamma_{j} M_{0}}-\frac{H_{0}^{(e)}}{M_{0}}-\beta_{j}\right)} .
\end{gathered}
$$

The local dispersion relation for a thin nanotube (20) agrees with the dispersion relation for a thin ferromagnetic film and for a thin cylindrical nanowire, see, e.g., [8, 31]. This implies that the spin wave pattern is similar for small enough (by one or two dimensions) ferromagnetic nanoobjects.

Let us make numerical evaluations for the spin wave frequency given by (8) in the absence of external magnetic field, assuming that the local longitudinal wave number is restricted, on the one hand, by the nanotube length (which makes unities or tens of micrometers for typical nanotubes), and, on the other hand, by the continuous medium approximation (so that the wavelength must be considerably greater than the interatomic distance) and by the exchange interaction length (has the order of several nanometers for typical ferromagnetics). Similar restrictions are imposed on the transverse wave number, with the addition that the latter vanishes when $n=0$. Thus, both the local longitudinal wave number $k_{j \|}$ and the total wave number $k_{j}$ for a typical nanotube change from $10^{2} \mathrm{~cm}^{-1}$ to $10^{6} \mathrm{~cm}^{-1}$ by the order of magnitude. For a typical ferromagnetic nanotube $\beta_{j} \sim 1, \alpha_{j} \sim 10^{-12} \mathrm{~cm}^{-2}$, so for a nanotube sections consisting of material with the gyromagnetic ratio $\gamma_{j}=$ $10^{7} \mathrm{~Hz} / \mathrm{Gs}$ and the saturation magnetization $M_{0}=10^{3}$ Gs spin wave frequency $\omega$, according to (8), has the order of magnitude of $10^{10} \mathrm{~Hz}$ throughout all the wave numbers range.

As one can see from (11), the transverse wave number increases when the nanotube thickness is decreased. (Note that expression for the transverse wave number spectrum we obtained for a periodically layered nanotube is true for a uniform nanotube as the sections' parameters doesn't enter it.) As typical nanotubes are thin, this fact puts a limitation on the mode number $n$ (because of the transverse wave number $k_{\perp}$ exchange length limitations). In particular, when the nanotube thickness is small compared to the exchange length $\left(b-a<<l_{e x}\right)$, the magnetization is uniform on the nanotube thickness, so we can put $k_{\perp}=0$; therefore, for very thin tubes $\left(b-a<l_{e x}\right)$ only zero transverseangular mode is possible $(n=0)$. For typical nanotubes that have a thickness of tens of nanometers, the number of possible transverseangular modes has an order of $(b-a) / l_{e x} \sim 10$.

From the expression (19) for the quasiwave number spectrum in an entire nanotube we can see that when the spin wave wavelength is much greater than the characteristic size of the nanotube inhomogeneities, an effective wave number in the whole nanotube can be found by averaging the local wave numbers in the tube sections. Moreover, for a thin nanotube (the thickness $b-a$ is small compared to the characteristic length of the exchange interaction, so relation (8) transforms into (20)) expres- 
sion (19) for the effective wave number agrees with the dispersion relation obtained in [31] for a thin periodically structured cylindrical nanowire:

$$
K=\sqrt{\frac{1}{\bar{\alpha}}\left(\frac{\omega}{\bar{\gamma} M_{0}}-\frac{H_{0}^{(e)}}{M_{0}}-\beta\right)},
$$

where $\bar{\gamma}=\left(\frac{\gamma_{1}^{-1} d_{1}+\gamma_{2}^{-2} d_{2}}{d_{1}+d_{2}}\right)^{-1}, \bar{\beta}=\frac{\beta_{1} d_{1}+\beta_{2} d_{2}}{d_{1}+d_{2}}$. As one can see, this expression agrees also with expression (20) for a local wave number of a thin nanotube after the ferromagnetic parameters that enter (20) are replaced with averaged parameters of the entire nanotube. So, in this case the spin wave in a periodically structured nanotube is described by the same dispersion relation as the wave in a uniform nanotube, with the difference that instead of the parameters of one ferromagnetic the dispersion relation contains effective (averaged) parameters of two nanotube ferromagnetics.

\section{Conclusions}

In this paper, we have investigated spin waves in a periodically layered ferromagnetic nanotube comprised of alternating layers of two kinds of ferromagnetic. We have studied the case when both ferromagnetics have "easy axis" type. We have obtained the radial (transverse) wave number spectrum and the longitudinal quasi-wave number spectrum for the spin wave in such system as well as the local dispersion relation (in the nanotube sections). We have shown that the local dispersion relation for a cylindrical nanotube passes (for a thin nanotube) into the known dispersion relation for a thin ferromagnetic nanowire. For a nanotube that is thin compared to the characteristic exchange interaction length, we have shown that the local dispersion relation becomes quadratic in the wave number. We have also shown that for a nanotube that is thin so that its thickness is much less than its inner radius (which is true for a typical nanotube) the wave number levels become equidistant, and the distance between them is inversely proportional to the nanotube thickness.

We have shown that for spin waves in typical nanotubes - both continuous and periodically layered - only the first $N$ transverse-angular modes can be excited, where the number $N \sim(b-a) / l_{e x}$ (here $a$ and $b$ are the inner and the outer radii of the nanotube, respectively, $l_{e x}$ is the characteristic exchange interaction length) has an order of 10 for a typical nanotube.

We have simplified expression for the quasi-wave number spectrum for the case when spin wave wavelength is much greater than the spatial period of the system (the "effective medium" limit), obtaining more simple and physically clear expression. Analyzing this expression, we have shown that the spin wave in such system is described by the same dispersion relation as the wave in a uniform nanotube (after averaging the local longitudinal wave number by the nanotube sections, or - for a thin nanotube substituting effective averaged parameters of two ferromagnetics into the dispersion relation for a uniform nanotube).

\section{References}

1. V.V.Kruglyak, S.O. Demokritov, D.Grundler, J.Phys. D :Appl. Phys., 43, 264001 (2010).

2. R.P.van Stapele, F.J.A.M.Greidanus, J.W.Smits, J.Appl. Phys., 57, 1282 (1985).

3. B.A.Kalinikos, N.G.Kovshikov, A.N.Slavin, J. Appl. Phys., 69, 5712 (1991).

4. M.Bauer, O.Buttner, S.O.Demokritov et al., Phys. Rev. Lett., 81, 3769 (1998).

5. K.Yu.Guslienko, A.N.Slavin, J.Appl.Phys., 87, 6337 (2000).

6. F.G.Aliev, J.F.Sierra, A.A.Awad et al., Phys. Rev. B, 79, 174433 (2009).

7. J.Jorzick, S.O.Demokritov, C.Mathieu et al., Phys.Rev. B, 60, 15194 (1999).

8. R.Arias, D.L.Mills, Phys.Rev.B, 63, 134439 (2001).

9. .R.Skomski, M.Chipara, D.J.Sellmyer, J.Appl. Phys., 93, 7604 (2003).

10. P.C.Fletcher, C.Kittel, Phys.Rev., 120, 2004 (1960).

11. S.M.Cherif, Y.Roussigne, C.Dugautier et al., J. Magn. Magn. Mater., 222, 337 (2000).

12 U.Ebels, J.-L.Duvail, P.E.Wigen et al., Phys. Rev. B, 64, 144421 (2001).

13 A.Encinas-Oropesa, M.Demand, L.Piraux et al., Phys. Rev. B, 63, 104415 (2001).

14 M.R.Freeman, B.C.Choi, Science, 294, 1484 (2001).

15 Y.Zhang, H.Dai, Appl.Phys. Lett., 77, 3015 (2000).

16 Y.Zhang, N.W.Franklin, R.J.Chen et al., Chem. Phys. Lett., 35, 331 (2000).

17. K.Nielsch, F.J.Castano, C.A.Ross et al., J. Appl. Phys., 98, 034318 (2005).

18 Y.C.Sui, R.Skomski, K.D.Sorge, D.J.Sellmyer, Appl.Phys. Lett., 84, 1525 (2004).

19. K.Nielsch, F.J.Castano, S.Matthias et al., Adv. Engin. Mater., 7, 217 (2005).

20. P.Landeros, S.Allende, J.Escrig et al., Appl. Phys. Lett., 90, 102501 (2007). 
21. Z.K.Wang, H.S.Lim, H.Y.Liu et al., Phys. Rev. Lett., 94, 137208 (2005).

22. W.Sharif, S.Shamaila, M.Ma et al., Appl.Phys. Lett., 92, 032505 (2008).

23. X.F.Salem, X.F.Searson, K.W.Leong, Nat. Mater., 2, 668 (2008).

24. K.W.Berry, K.W.Curtis, J.Phys.D:Appl. Phys., 36, R198 (2003).

25. H.Leblond, V.Veerakumar, Phys.Rev.B, 70, 134413 (2004).

26. V.Gonzalez, P.Landeros, P.Nunez, J.Magn. Magn. Mater., 322, 530 (2010).

27. M.Inoue, H.Uchida, K.Nishimura, P.B.Lim, $J$. Mater. Chem., 16, 678 (2006).

28. M.Inoue, R.Fujikawa, A.Baryshev et al., $J$. Phys. D :Appl. Phys., 39, R151 (2006).

29. M.Inoue, K.Arai, T.Fujii et al., J.Appl.Phys., 85, 5768 (1999).
30. H.Kato, T.Matsushita, A.Takayama et al., Opt.Commun., 219, 271 (2003).

31. V.V.Kruglyak, R.J.Hicken, A.N.Kuchko et al., J.Appl.Phys., 98, 014304 (2005).

32. V.V.Kruglyak, A.N.Kuchko, Physica B, 339, 130 (2003).

33. S.A.Nikitov, P.Tailhades, C.S.Tsai, J.Magn. Magn. Mater., 236, 320 (2001).

34. C.Elachi, IEEE Trans. Magn., MAG-11, 36 (1975).

35. Yu.V.Gulyaev, A.A.Nikitov, Doklady Physics, 46, 687 (2001).

36. A.I.Akhiezer, V.G.Bar'yakhtar, S.V.Peletminskiy, Spin Waves, North-Holland, Amsterdam (1968).

37. V.V.Kruglyak, A.N.Kuchko, V.I.Finokhin, Phys. Solid State, 46, 867 (2004). 